\title{
Synchrotron X-Ray Study of the Thickness Dependence of the Phase Diagram of Thin Liquid- Crystal Films
}

\section{Citation}

Collett, Jeffrey, Peter S. Pershan, Eric B. Sirota, and L. B. Sorensen. 1984. Synchrotron x-ray study of the thickness dependence of the phase diagram of thin liquid-crystal films. Physical Review Letters 52(5): 356-359.

\section{Published Version}

doi:10.1103/PhysRevLett.52.356

\section{Permanent link}

http://nrs.harvard.edu/urn-3:HUL.InstRepos:10361602

\section{Terms of Use}

This article was downloaded from Harvard University's DASH repository, and is made available under the terms and conditions applicable to Other Posted Material, as set forth at http:// nrs.harvard.edu/urn-3:HUL.InstRepos:dash.current.terms-of-use\#LAA

\section{Share Your Story}

The Harvard community has made this article openly available.

Please share how this access benefits you. Submit a story.

\section{Accessibility}




\title{
Synchrotron X-Ray Study of the Thickness Dependence of the Phase Diagram of Thin Liquid-Crystal Films
}

\author{
Jeffrey Collett, (a) P. S. Pershan, Eric B. Sirota, and L. B. Sorensen ${ }^{\text {(b) }}$ \\ Division of Applied Sciences, Harvard University, Cambridge, Massachusetts 02138
}

(Received 7 October 1983)

\begin{abstract}
The phase diagram of freely suspended thin films of heptyloxybenzylidene-heptylaniline shows dramatic changes for thicknesses below 22 layers. The most surprising feature of the phase diagram is the inclusion of two phases lacking long-range crystalline order (smectic- $F$ and hexatic $-B$ phases) between two crystalline phases (crystalline smectic $B$ and smectic $G$ ). Neither the smectic $F$ nor the hexatic $B$ occurs in bulk samples. Between sixteen and ten layers the width, in temperature, of the hexatic- $B$ phase increases.

PACS numbers: 64.70.-p, 61.30.-v
\end{abstract}

Freely suspended liquid-crystal films provide one system for studying the crossover from three to two dimensions. ${ }^{1}$ Interest in this problem has been stimulated by suggestions that transitions in three-dimensional (3D) smectic liquid crystals may be related to transitions within individual 2D layers $\mathrm{s}^{2,3}$ and by the development of a detailed theory of 2D melting. ${ }^{4}$ The most experimentally accessible prediction of the theory of $2 \mathrm{D}$ melting is the existence of an intermediate hexatic phase with short-range positional order but with quasilong-range bond-angle orientational order. A recent study of melting in variable-thickness films of $\overline{14} 55$ has shown the existence of an intermediate hexatic phase in a three-layer film which is not observed in bulk. ${ }^{5}$ In this study we report the existence of a smectic-F phase (hexatic layers with a molecular tilt) in films as thick as 22 layers and the appearance of a hexatic- $B$ phase at sixteen layers in a system which has no such phases in bulk samples.

We have examined freely suspended films of 4 - $n$-heptyloxybenzylidene $4-n$-heptylaniline (70.7) varying in thickness from 5 to 22 layers. Previous work ${ }^{6}$ has shown that thick films (>500 layers) of 70.7 exhibit rich structure with a series of three transitions involving restacking of the layers and subtle changes in the intralayer packing. In order of decreasing temperature, the observed crystal structures are hexagonal-closepacked (hcp), orthorhombic face-centered (ortho$\boldsymbol{F})$, monoclinic $C$-centered (mono- $C$ ), and simple hexagonal (hex- $A A$ ) structures. The last three of these have a one-dimensional modulation of the smectic layers which has a wavelength of 95 $\AA$, a wave vector in the plane of the molecular layers directed along a reciprocal-lattice direction, and a polarization normal to the plane of the smectic layers. Immediately below the hex- $A A$ structure is a smectic- $G$ phase, a monoclinic structure with the long axes of the molecules tilted by 24 deg with respect to the layer normal. The projection of the long axes of the molecules onto the 2D triangular in-plane lattice is directed halfway between nearest neighbors. ${ }^{7}$ This complex behavior is undoubtedly due to a combination of intralayer and interlayer effects. The present study was undertaken to clarify the origin of the restacking transitions and to look for the effects of reduced dimensionality.

$\mathrm{X}$-ray diffraction studies were carried out on the triple-axis spectrometer installed on beam line VII-2 at the Stanford Synchrotron Radiation Laboratory. ${ }^{8}$ A pair of asymmetrically cut Ge[111] crystals were used in the double-crystal monochromator. Low-resolution measurements were made with a pyrolytic graphite [002] analyzer which resulted in a longitudinal resolution of $\Delta Q=22 \times 10^{-3} \AA^{-1}$. Higher-resolution measurements were done with a $\mathrm{LiF}[200]$ analyzer which provided a longitudinal resolution of $\Delta Q=3.8$ $\times 10^{-3} \AA^{-1}$. In both cases a 5-mrad horizontal divergence of the input beam was accepted by the focusing mirror. The films were oriented with the normal to the smectic layers always in the scattering plane. Scans were done along $Q_{x y}$, parallel to the planes of the smectic layers, or along $Q_{z}$, normal to the layers. A rotation angle $\chi$, about an axis normal to the smectic layers, specified the direction of $Q_{x y}$ within the smectic layers.

We find that the phase sequence of 70.7 undergoes dramatic changes as a function of film thickness. Part of the thickness-temperature plane of the phase diagram is shown in Fig. 1. The measured points are shown together with speculative phase boundaries. The most striking feature of this phase diagram is the inclusion of phases lacking long-range crystalline order (smectic- $F$ and hexatic $-B$ phases) between the crystalline- $B$ and smectic- $G$ phases, both of which are crystalline. For thicknesses below 22 layers, we find 


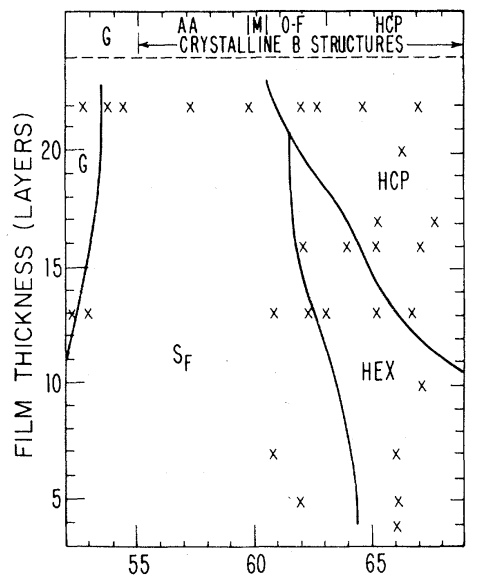

(a) TEMPERATURE $\left({ }^{\circ} \mathrm{C}\right)$

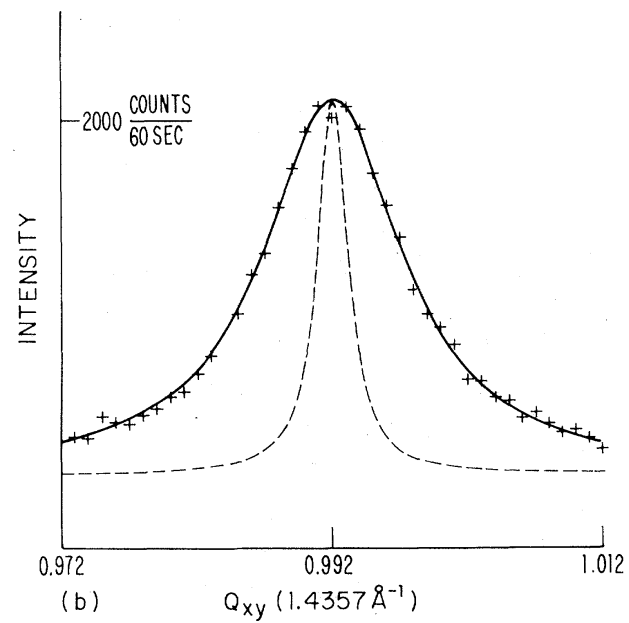

(b) $\quad Q_{x y}\left(1.4357 \AA^{-1}\right)$

FIG. 1. (a) Thickness vs temperature phase diagram for 7O.7. The phases are denoted by $G$, crystalline $G$; $A A$, hex- $A A$ crystalline $B ; M$, mono- $C$ crystal; $O-F$, ortho- $F$; HCP, hcp; $S_{F}$, smectic $F$; and HEX, hexatic $B$. Measured points are marked by crosses with speculative phase boundaries shown. (b) $Q_{x y}$ scan in hexatic- $B$ phase of thirteen-layer film at $T=65.07^{\circ} \mathrm{C}$. The solid line is a fit by the sum of a Lorentzian and a constant background. The dashed line is the spectrometer resolution displayed on the same background.

that a smectic- $F$ phase occupies the temperature region corresponding to the hex- $A A$ and mono- $C$ phases in bulk samples. As the sample thickness is reduced to sixteen layers, a hexatic- $B$ phase appears between the smectic-F and the crystalline $-B$ phases. Between sixteen and ten layers the hexatic $-B$ phase gradually replaces the crystalline- $B$ phase. Below ten layers the crystalline- $B$ phase seems to disappear.

All of the four phases (crystalline $B$, hexatic $B$, smectic $F$, and smectic $G$ ) observed in thin films are observed in thirteen-layer films. Figures $1(\mathrm{~b})$ and 2 illustrate the interlayer and intralayer correlations for a thirteen-layer film. Figure 2(a) shows a $Q_{z}$ scan in the crystalline- $B$ phase at $T=67.4^{\circ} \mathrm{C}$. There are definite peaks at $Q_{z}= \pm \frac{1}{2}$ but no peak at $Q_{z}=0$. The $Q_{z}=0$ peak is more difficult to observe because the theoretical intensity is only $\frac{1}{3}$ that of the $Q_{z}= \pm \frac{1}{2}$ peaks and stacking faults reduce its relative intensity even further. ${ }^{9}$

At $65.5^{\circ} \mathrm{C}$ there is a transition to a hexatic $-B$ phase. This has the properties theoretically predicted ${ }^{3}$ and observed in other smectic materials. ${ }^{10}$ These properties include a finite intralayer correlation length, a lack of interlayer correlations, and the presence of bond-angle orientation order. Figure $1(\mathrm{~b})$ shows a $Q_{x y}$ scan at $\boldsymbol{Q}_{\boldsymbol{z}}=0$ which has a width corresponding to a correlation length of $130 \AA$. This is similar to other hexatic- $B$ materials and is much longer than the correlation lengths observed in the smectic-
$A$ and $-C$ phases of 70.7. Figure 2(b) shows a $Q_{z}$ scan through the peak of the scan shown in Fig. 1(b). This scan shows a single broad peak with full width at half maximum of $1.7(2 \pi / d)$. This width is similar to the width expected from the molecular form factor and indicates that there are no significant interlayer correlations. Finally, $\chi$ scans in this phase show considerable struc-

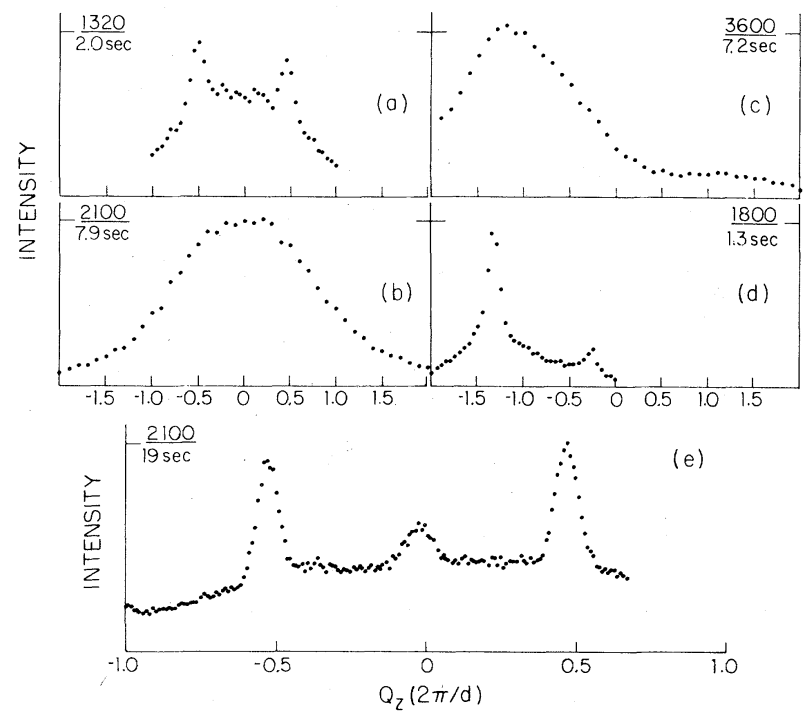

FIG. 2. $Q_{\boldsymbol{Z}}$ scans: (a) thirteen-layer crystalline $B$ at $T=67.4^{\circ} \mathrm{C}$, (b) thirteen-layer hexatic $B$ at $T=62.8^{\circ} \mathrm{C}$, (c) thirteen-layer smectic $F$ at $T=60.8^{\circ} \mathrm{C}$, (d) thirteenlayer crystalline $G$ at $T=51.2^{\circ} \mathrm{C}$, and (e) seventeenlayer crystalline $B$ at $T=67.6^{\circ} \mathrm{C}$. 
ture although single domains were not observed and the domain structure drifted with time.

At $61{ }^{\circ} \mathrm{C}$ there is an abrupt transition to a smectic- $F$ phase, which is a hexatic phase with long axes of the molecules tilted with respect to the normal to the smectic layers. The correlation length is similar to the hexatic $-B$ phase and $\chi$ scans also show structure. The $Q_{z}$ scan in Fig. 2 (c) differentiates this phase from the hexatic- $B$ phase; the width of the broad peak is similar to the scan in the hexatic phase [Fig. 2(b)] but the center of the peak is displaced to nonzero $Q_{z}$. This is because the molecular form factor has its maximum in the direction normal to the long axis of the molecule. From the position of the peak the molecular tilt is estimated to be $20 \mathrm{deg}$ in the smectic- $F$ phase.

At $T=51.6^{\circ} \mathrm{C}$ the thirteen-layer film crystallized into a smectic- $G$ phase. Figure 2(d) shows a $\boldsymbol{Q}_{\boldsymbol{z}}$ scan in this phase which shows sharp peaks characteristic of the monoclinic structure. The $Q_{x y}$ scans in this phase show no excess width with graphite resolution, implying that the intralayer ordering is long ragne.

In films with thicknesses greater than ten molecular layers, the first phase which appears at temperatures below the smectic- $C$ phase is the crystalline- $B$ hcp structure. This phase is characterized by resolution-limited peaks in the $\boldsymbol{Q}_{z}$ $=0$ plane. When $Q_{x y}$ is scanned through one of these in-plane peaks, results similar to the dashed line in Fig. 1(b) are obtained. Peaks appear at integer and half-integer values of $Q_{\boldsymbol{z}}$ (measured in units $2 \pi / d$, where $d$ is the molecular length). Figure 2(e) shows a scan in a seventeen-layer film; here the intensities of the peaks are in the 3:1:3 ratio expected for the hcp structure. As the transition to the hexatic-B phase is approached, the intensity of the peak at $\boldsymbol{Q}_{z}=0$ drops relative to the half-order peaks and the intensity of the thermal diffuse background grows relative to the peaks [Fig. 2(a)]. The widths of the peaks in these $Q_{z}$ scans are greater than the finite size limit. The resolution of the present experiments was not high enough to distinguish an ortho- $F$ structure from a faulted hep structure.

Films thinner than ten layers appear to have no crystalline- $B$ phase, only the hexatic- $B$ and smectic-F phases. We have not yet measured very thin films in the region of the smectic- $F$ to smectic- $G$ transition, but we expect the $G$ phase to occur below the $F$. There is one difference between the data taken on five-layer films and those obtained from thirteen-layer films. The hexatic- $B$ and smectic- $F$ phases in the five-layer films show no structure in $\chi$ scans. We identify these phases by the intralayer correlation lengths obtained from $Q_{x y}$ scans and from the interlayer correlations measured with the $Q_{z}$ scans. The loss of measurable orientational order in very thin films of materials which have bulk hexatic$B$ phases has been noted in other experiments. ${ }^{11}$ This is assumed to be related to both static defects and the quasi-long-range nature of the orientational ordering in the two-dimensional hexatic. ${ }^{11,12}$ The interlayer correlations measured in the smectic- $F$ phase for the five-layer film clearly rule out the possibility of a smectic$C$ phase with unusually long intralayer correlation lengths.

The nature of this transformation of the system from a crystalline solid to a hexatic with short-range positional order is not understood. At present we do not know at what thickness the crystalline phases characteristic of bulk 70.7 are replaced by the smectic-F phase. The fact that a tilted hexatic phase replaces the crystalline structures supports the suggestion that molecular tilt is involved in the restacking transitions observed in the bulk. ${ }^{6}$ The crossover from the crystalline phases to the smectic- $F$ phase is probably due to a competition between bulk and surface energies. As the thickness of the sample is reduced, the contribution of the surface term to the total free energy becomes more important and ultimately results in the instability of the crystalline structures. The free-energy difference between the crystalline structures and the bulk smectic- $F$ structure may be very small, since materials such as 50.6 have a phase sequence in bulk (crystalline $B-$ smectic $F$-smectic $G$ ) which is identical to the one observed in 22-layer films of 70.7.7

This study reveals an amazing richness of structure which poses a new set of questions to be answered. A systematic study of crystalline$B$ line shapes as a function of thickness may give further insight into the details of the crystal to hexatic transition. Preliminary data suggest that the smectic- $F$ to smectic- $G$ transition in the thin films may be a second-order transition. An understanding of this system may lead to the understanding of other bulk liquid-crystal systems such as 50.6 which have a liquidlike phase sandwiched between two crystalline phases. ${ }^{7}$ Further studies of these systems promise to enhance our understanding of both three-dimensional layered systems and the role of reduced dimensionality. 
This research was supported by the National Science Foundation under Grant No. NSF-DMR8212189. The work reported herein was performed at the Stanford Synchrotron Radiation Laboratory, which is supported by the U. S. Department of Energy, Office of Basic Energy Sciences; the National Science Foundation, Division of Materials Research; and the National Institutes of Health, Biotechnology Resource Program, Division of Research Resources. We wish to acknowledge the assistance of the staff at the Stanford Synchrotron Radiation Laboratory, especially T. Porter, in carrying out these measurements. We would also like to thank R. Pindak and D. E. Moncton for helpful discussions of the experiments.

(a) Present address: IBM Research Laboratory, Yorktown Heights, N.Y. 10598.

(b) Present address: Department of Physics FM-15, University of Washington, Seattle, Wash. 98195.

${ }^{1}$ D. E. Moncton and R. Pindak, Phys. Rev. Lett. $\underline{43}$, 701 (1979).
${ }^{2}$ P. G. de Gennes and G. Sarma, Phys. Lett. 38A, 219 (1972); B. A. Huberman, D. M. Lublin, and S. Doniach, Solid State Commun. 17, 485 (1975).

${ }^{3}$ R. J. Birgeneau and J. D. Litster, J. Phys. (Paris), Lett. 39, L399 (1978).

${ }^{4}$ D. R. Nelson and B. I. Halperin, Phys. Rev. B $\underline{19}$, 2457 (1979).

${ }^{5}$ D. E. Moncton, R. Pindak, S. C. Davey, and G. S. Brown, Phys. Rev. Lett. 49, 1865 (1982).

${ }^{6} \mathrm{~J}$. Collett, L. B. Sorensen, P. S. Pershan, J. D. Litster, R. J. Birgeneau, and J. Als-Nielsen, Phys. Rev. Lett. 49, 553 (1982).

${ }^{7}$ A. J. Leadbetter, M. A. Mazid, and R. M. Richardson, in Liquid Crystals, edited by $\mathrm{S}$. Chandrasekhar (Cambridge Univ. Press, London, 1980), pp. 65-79; J. W. Goodby, G. W. Gray, A. L. Leadbetter, and M. A. Mazid, J. Phys. (Paris) 41, 591 (1980).

${ }^{8}$ D. E. Moncton and G. S. Brown, Nucl. Instrum. Methods 208, 579 (1983).

${ }^{9}$ A. Gunier, X-Ray Diffraction (Freeman, San Francisco, 1963).

${ }^{10}$ R. Pindak, D. E. Moncton, J. W. Goodby, and S. C. Davey, Phys. Rev. Lett. $\underline{46}, 1135$ (1981).

${ }^{11} \mathrm{~J}$. Budai, S. C. Davey, R. Pindak, and D. E. Moncton, Stanford Synchrotron Radiation Laboratory Report No. 83101, 1983 (unpublished), p. VII-102.

${ }^{12}$ T. F. Rosenbaum, S. E. Nagler, P. M. Horn, and R. Clark, Phys. Rev. Lett. 50, 1791 (1983). 